\title{
Effect of amlodipine on the manifestations of chronic insomnia in hypertensive patients with type 2 diabetes mellitus
}

\author{
Ganna Isayeva $\odot^{1}$, Olena Buriakovska $\odot^{1}$, Anna Shalimova $\oplus^{1,2}$ \\ 'Government Institution 'L.T. Malaya Therapy National Institute of the National Academy of Medical Sciences of Ukraine', Kharkiv, Ukraine \\ ${ }^{2}$ Kharkiv National Medical University, Kharkiv, Ukraine
}

\begin{abstract}
Background: The aim of the study was to assess the association between antihypertensive therapy and the manifestations of insomnia in patients with arterial hypertension (AH) and type 2 diabetes mellitus (T2DM).

Material and methods: The study included 120 hypertensive patients with and without T2DM (among them there were 60 patients with insomnia). The study consisted of three stages. The first stage was conducted as a cross-sectional study, during which an association was established between different antihypertensive products and the presence of insomnia in the study population. The second and third stages were a prospective study, during which a modification of the therapy to reduce the manifestations of insomnia was performed.

Results: It was found that patients receiving amlodipine in the combination antihypertensive therapy had insomnia manifestations much less frequently as compared to indapamide. A statically significant decrease in blood pressure (BP) and a higher proportion of patients with target BP were observed in both groups. Replacement of indapamide with amlodipine was shown to improve sleep quality. Thus, the number of patients with insomnia significantly decreased in both groups. After correction of antihypertensive therapy after 12 months, all patients were assessed for sleep disorders. The incidence of insomnia was found to be significantly reduced in both groups after changing therapy from indapamide to amlodipine.

Conclusions: Correction of antihypertensive therapy, namely the replacement of indapamide with amlodipine, contributes to an improvement in BP, quality of life, and a reduction in the proportion of patients with insomnia.
\end{abstract}

Key words: insomnia; arterial hypertension; type 2 diabetes mellitus; amlodipine

$$
\text { Arterial Hypertens. 2020, vol. 24, no. 4, pages: 192-199 }
$$

DOI: $10.5603 /$ AH.a2020.0026

\section{Introduction}

The association between insomnia and cardiovascular diseases has been proven in a large number of works [1-4]. According to Taylor [5], 40\% of patients with diseases of the cardiovascular system have problems with the quality or duration of sleep. A high prevalence of insomnia among patients with hypertension and its effect on the course of hypertension was demonstrated in a systemic review of Jarrin [6]. Li [7] found that difficulties in maintaining sleep and early morning awakenings significantly increased the risk of developing hypertension. Fernamdez-Mendoza [8] confidently demonstrated that insomnia was an independent risk factor for developing hypertension. A higher risk of developing hypertension in people

Address for correspondence: Prof. Anna Shalimova, Department of Internal Medicine No 1, Kharkiv National Medical University,Kharkiv, Ukraine; e-mail: anna.shalimova83@gmail.com 
with insomnia was also found in study of Lin [9]. For instance, according to these authors, patients with insomnia have a $21 \%$ higher risk of developing hypertension compared with those who have quality sleep [9]. At the same time, Wang [10] found that insomnia is much more common in patients with hypertension (36.35\%) compared with the general population (28.9\%). Similar data were presented in a study of Bathgate [11], who found that almost $36.9 \%$ of patients with hypertension had some form of insomnia. A study, which included $21.678 \mathrm{pa}-$ tients with a medical history of stroke, demonstrated that those with hypertension were more likely to take sleeping pills [12]. The presented literature data show that insomnia is both a risk factor for hypertension and often accompanies it. It should be noted that a patient with both diseases can live quite a long time and there is a problem of finding the optimal therapy for this group of patients.

At the same time, data on the effect of antihypertensive therapy on the quality and duration of sleep are scarcely presented in the modern literature.

The aim of the study was to assess the association between antihypertensive therapy and the manifestations of insomnia in patients with hypertension and type 2 diabetes mellitus.

\section{Material and methods}

\section{General characteristics and design of the study}

The study consisted of three stages. The first stage was conducted as a cross-sectional study, during which an association was established between different antihypertensive products and the presence of insomnia in the study population. The second and third stages were a prospective study, during which a modification of the therapy to reduce the manifestations of insomnia was performed. The study design is presented in Figure 1.

The inclusion criteria were age over 45 years, presence of hypertension. The diagnosis of hypertension was established according to the 2018 ESC/ESH Guidelines for the management of arterial hypertension [13]. The diagnosis of type 2 diabetes mellitus was established according to the Unified Clinical Protocol of Primary and Secondary (Specialized) Medical Care: Type 2 Diabetes (Order of the Ministry of Health No. 1118 of 21.12.2012) [14].

The study did not include patients who had cardiovascular events (myocardial infarction, stroke), patients with heart failure or with an ejection fraction of less than $45 \%$, with resistant arterial hypertension, thyroid gland dysfunction, gastric ulcer and duodenal ulcer in the exacerbation phase, with concomitant diseases affecting the quality of life, patients with obesity grade III, with concomitant any active cancer, with depressive states (organic depressive disorder (F 06.32), recurrent depressive disorder (F 33.0-3) or a depressive episode of any severity (F 31.3-5, F 32.0-3), also a mixed anxiety and depressive disorder ( $\mathrm{F}$ 41.2) according to the criteria of the International Classification of Diseases 10th revision (ICD-10), with insomnia as a symptom of any mental illness, with concomitant therapy with glucocorticosteroids, antihypertensive drugs of central action (moxonidine, reserpine), beta-blockers, hypnotics, patients who confirmed eating at night, patients with obstructive sleep apnea syndrome.

During stage 2, all patients, who were diagnosed with sleep disorders, were enrolled in "Health Schools" for 3 months, after which the following were assessed: sleep disorders, quality of life, anthropometric, hemodynamic, and biochemical parameters. After finishing the "Health Schools", there were 56 patients with symptoms of insomnia in the general group, among whom 36 patients had combined hypertension and type 2 diabetes mellitus, and 20 of them had isolated hypertension. A detailed analysis of the antihypertensive therapy showed that in the group of combined hypertension and diabetes mellitus with insomnia symptoms, indapamide was taken by $78 \%$ of patients, while amlodipine by $22 \%$ $(p=0.05)$. The same trend was observed in the group of isolated hypertension with insomnia: indapamide was taken by $70.6 \%$ of patients, while amlodipine by $29.4 \%(p=0.05)$. Due to the fact that insomnia was less common among patients in both groups receiving amlodipine, indapamide therapy was replaced by amlodipine. Patients received this therapy for 12 months. Then, sleep disorders, quality of life index, hemodynamic parameters were re-assessed and the percentage of patients who reached the target blood pressure was calculated (Fig. 1).

\section{Assessment of sleep disorders}

The presence of insomnia was assessed using the criteria of the International Classification of Sleep Disorders, $3^{\text {rd }}$ Edition, which was developed by the International Academy of Sleep Disorders, 2014 [15]. The diagnosis of insomnia was established in the presence of the following criteria: $1-$ difficulty falling asleep or staying asleep continuously; 2 - availability of adequate sleeping conditions; 3 - day consequences of poor sleep quality [15]. The night apnea syndrome was detected by night monitoring with the use of a SOMNOcheck micro CARDIO device (2013, Germany). 


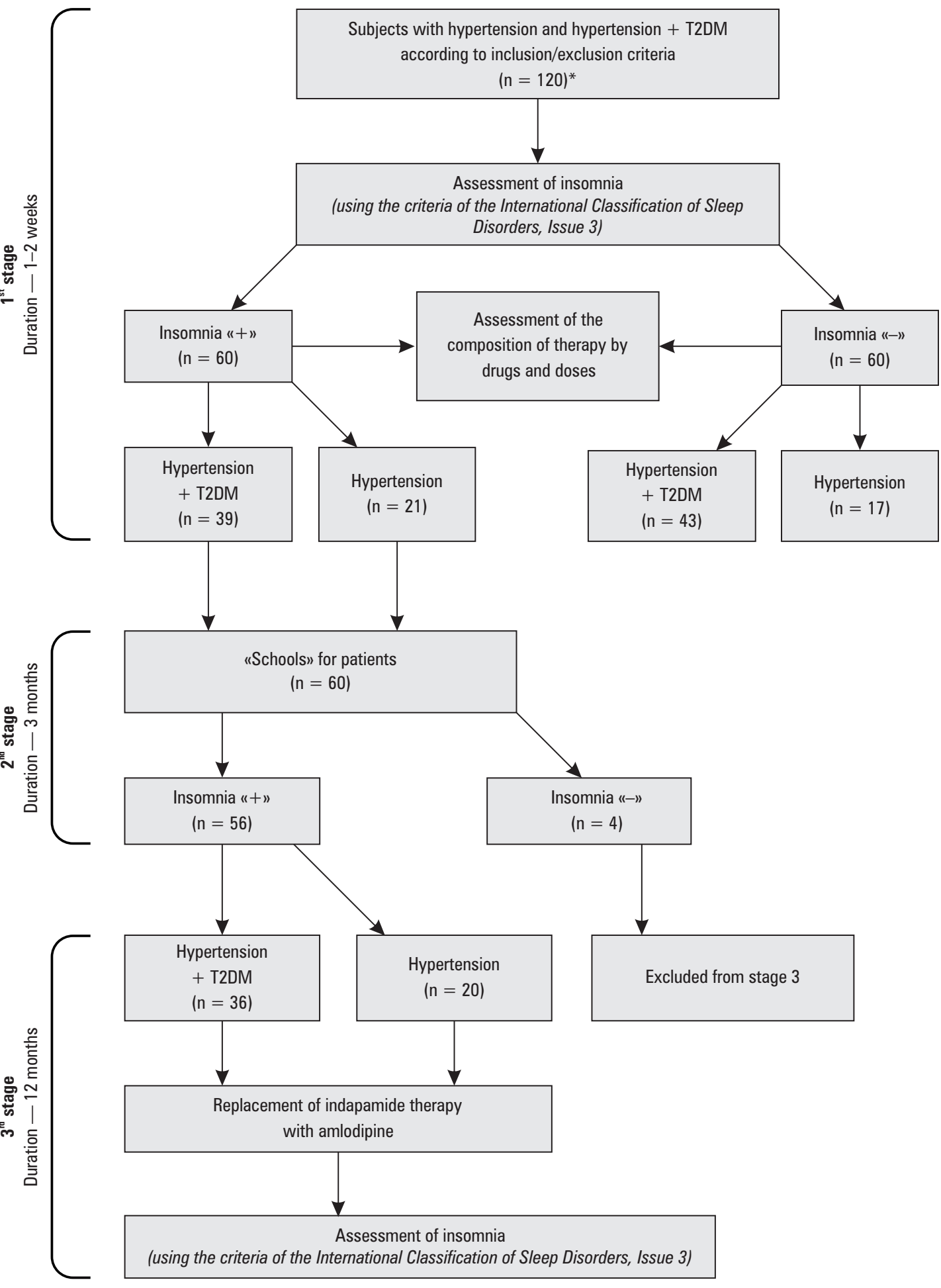

Figure 1. Design of the study. ${ }^{*} " »$ Insomnia is present; «-» Insomnia is absent. T2DM — type 2 diabetes mellitus 


\section{Instrumental examination}

Blood pressure was measured in accordance with the current recommendations of the European Society of Cardiology [13].

\section{Assessment of achievement of target indicators}

Achievement of target blood pressure levels and lipid metabolism values was assessed in accordance with current recommendations of the European Society of Cardiology [13].

\section{Therapy}

Patients were initially treated with an ACE inhibitor (ramipril) or sartan (valsartan), a diuretic (indapamide) and a calcium antagonist (amlodipine). All patients received atorvastatin as a lipid-lowering therapy and metformin as an antidiabetic therapy.

\section{Statistical data processing}

Statistical analysis was performed with the use of SPSS, version 17.0 (Chicago, IL. USA). The type of data distribution was assessed using the KolmogorovSmirnov method. With the normal distribution of continuous values, the data are presented as mean values (M) and standard deviation (SD), for quantitative values - the number (n) and frequency (\%). Data which did not meet the requirements of normal distribution were presented as medians and 25\% and $75 \%$ quartiles. The Student's t-test was used for comparing two independent samples corresponding to normal distribution. If the dependent samples did not meet the requirements of normal distribution, the comparison was performed using the MannWhitney test. Differences were considered as statistically significant if $\mathrm{p}<0.05$.

\section{Ethic aspects}

The study was approved by the Commission on Ethics and Deontology of the State Institution "L.T. Malaya National Institute of Therapy of the National Academy of Medical Sciences of Ukraine" on April 28,2017 . All participants of the study signed an informed consent, the aim and objectives of the study were previously clearly explained to them.

\section{Results}

Patients receiving amlodipine were found to have insomnia manifestations much less frequently (Fig. 2).

The therapy in groups after replacement of indapamide with amlodipine is shown in Figure 3.

A statically significant decrease in blood pressure (Tab. 1) and a higher proportion of patients with target blood pressure were observed in both groups (Fig. 4 and 5).

Replacement of indapamide with amlodipine was shown to improve sleep quality. Thus, the number of patients with insomnia significantly decreased in both groups.

After correction of antihypertensive therapy after 12 months, all patients were assessed for sleep disorders. The incidence of insomnia was found to be significantly reduced in both groups after changing therapy from indapamide to amlodipine ( $\mathrm{p}=0.05)$ (Fig. 7).

\section{Discussion}

Although insomnia is quite common in patients with essential hypertension and type 2 diabetes mellitus,

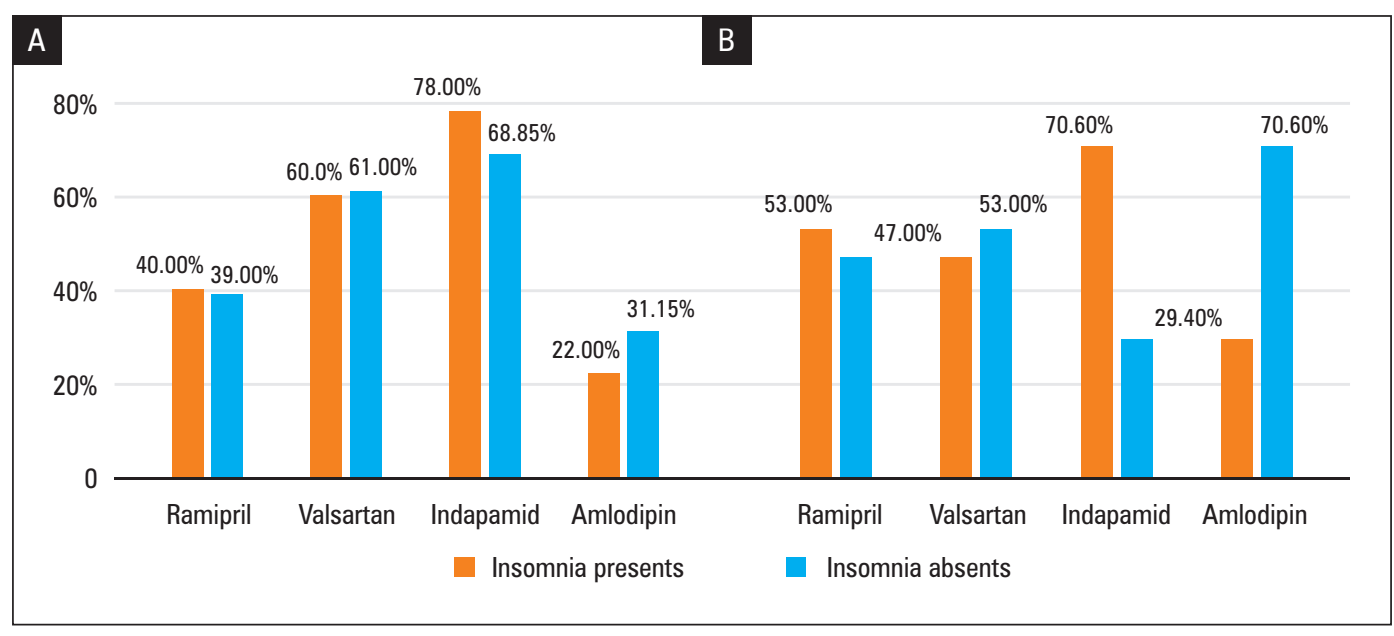

Figure 2. Medical therapy in groups with and without insomnia. A. Patients with hypertension + diabetes mellitus type 2. B. Patients with hypertension. Data presented as a proportion of patients (\%) treated with particular drug 


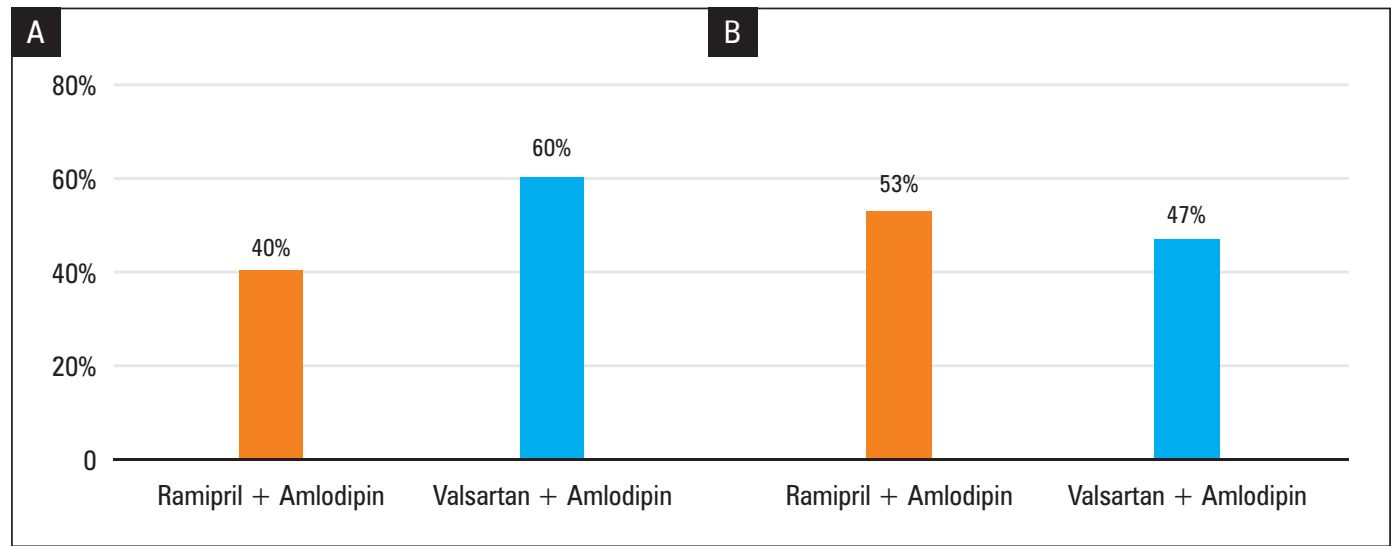

Figure 3. Medical therapy during third period of study (after replacement of indapamid to amlodipine). A. Patients with hypertension + type 2 diabetes mellitus. B. Patients with hypertension. Data presented as a proportion of patients (\%) treated with particular drug

Table 1. Dynamics of hemodynamic parameters of patients in groups with symptoms of insomnia before and after correction of therapy*

\begin{tabular}{|c|c|c|c|c|c|c|c|c|}
\hline \multirow{2}{*}{ Index } & \multicolumn{4}{|c|}{$\begin{array}{l}\text { Group } 1 \text { (hypertension + T2DM) } \\
\qquad(\mathrm{n}=36)\end{array}$} & \multicolumn{4}{|c|}{$\begin{array}{l}\text { Group } 2 \text { (hypertension) } \\
\qquad(\mathrm{n}=20)\end{array}$} \\
\hline & 1 stage & 2 stage & 3 stage & p & 1 stage & 2 stage & 3 stage & $p$ \\
\hline $\begin{array}{l}\text { SBP } \\
{[\mathrm{mm} \mathrm{Hg}]}\end{array}$ & $142.14 \pm 12.25$ & $138.71 \pm 7.25$ & $129.16 \pm 4.35$ & $\begin{array}{l}p^{1-2}=0.136 \\
p^{1-3}=0.001 \\
p^{2-3}=0.001\end{array}$ & $140.75 \pm 7.46$ & $137.35 \pm 14.16$ & $128.76 \pm 6.16$ & $\begin{array}{l}p^{1-2}=0.336 \\
p^{1-3}=0.001 \\
p^{2-3}=0.014\end{array}$ \\
\hline $\begin{array}{l}\text { DBP } \\
{[\mathrm{mm} \mathrm{Hg}]}\end{array}$ & $92.87 \pm 8.72$ & $88.87 \pm 7.62$ & $85.07 \pm 7.12$ & $\begin{array}{l}p^{1-2}=0.342 \\
p^{1-3}=0.001 \\
p^{2-3}=0.027\end{array}$ & $88.52 \pm 10.17$ & $85.72 \pm 7.36$ & $78.67 \pm 5.43$ & $\begin{array}{l}p^{1-2}=0.272 \\
p^{1-3}=0.003 \\
p^{2-3}=0.001\end{array}$ \\
\hline $\begin{array}{l}\text { Heart rate } \\
\text { (per minute) }\end{array}$ & $77.62 \pm 7.03$ & $74.90 \pm 7.91$ & $75.12 \pm 5.75$ & $\begin{array}{l}p^{1-2}=0.116 \\
p^{1-3}=0.087 \\
p^{2-3}=0.886\end{array}$ & $71.82 \pm 8.70$ & $68.62 \pm 6.13$ & $70.17 \pm 4.76$ & $\begin{array}{l}p^{1-2}=0.175 \\
p^{1-3}=0.536 \\
p^{2-3}=0.140\end{array}$ \\
\hline
\end{tabular}

T2DM — type 2 diabetes mellitus; SBP — systolic blood pressure; DBP — diastolic blood pressure. *Data presented in the view of the middle value and the standard deviation (M $\pm \sigma$ )

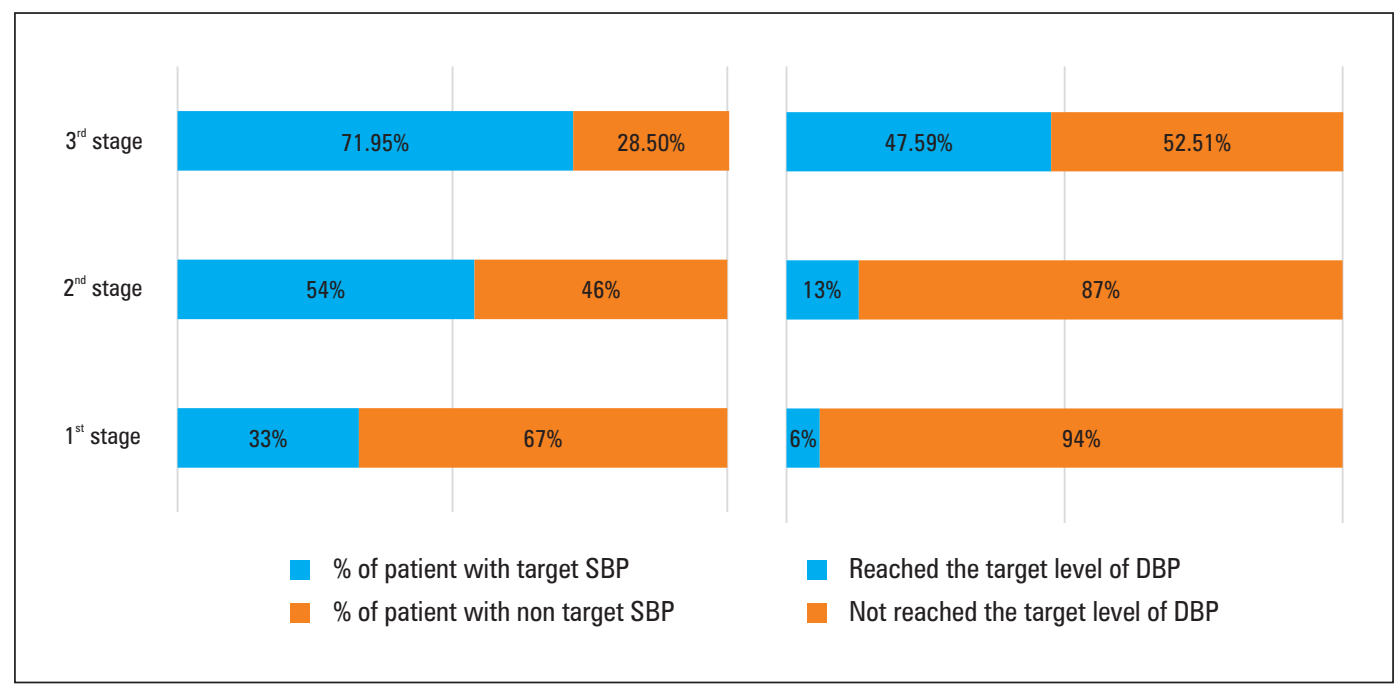

Figure 4. Number of patients with target systolic blood pressure (SBP) and diastolic blood pressure (DBP) in patients with hypertension and type 2 diabetes mellitus 


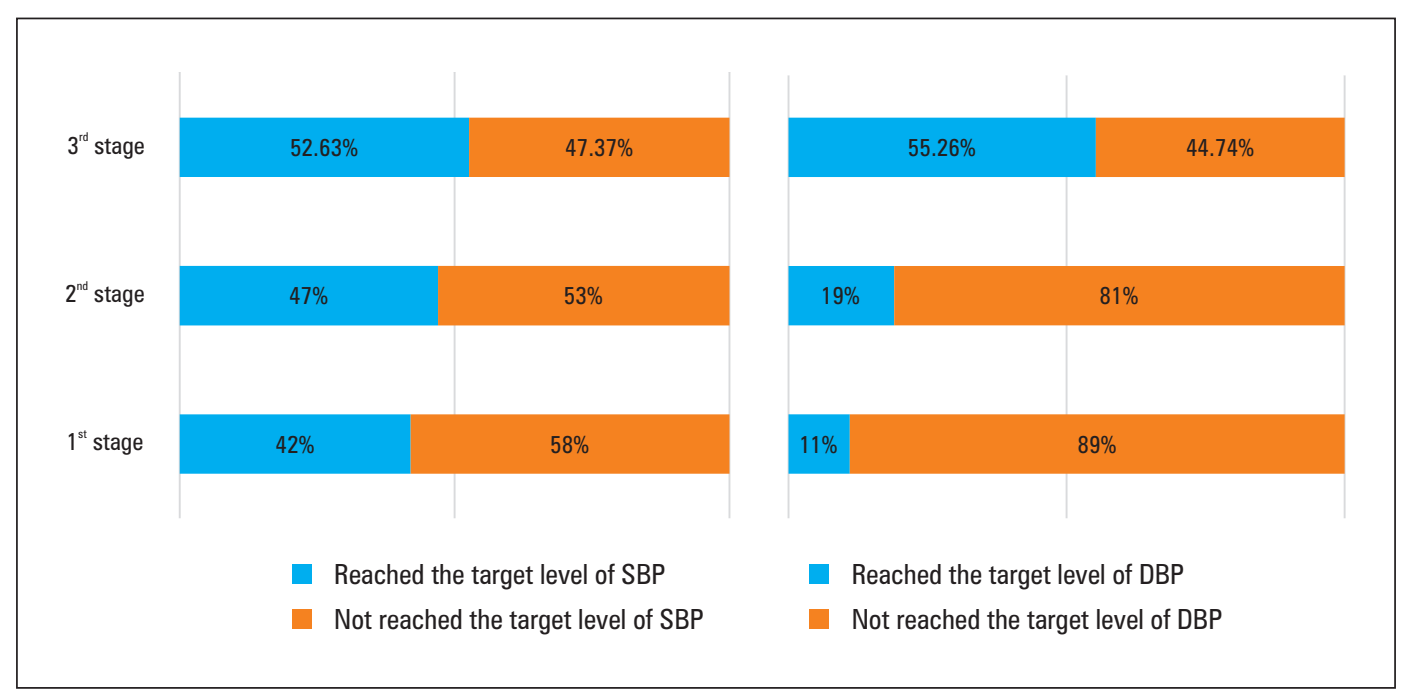

Figure 5. Number of patients with target systolic blood pressure (SBP) and diastolic blood pressure (DBP) in patients with hypertension

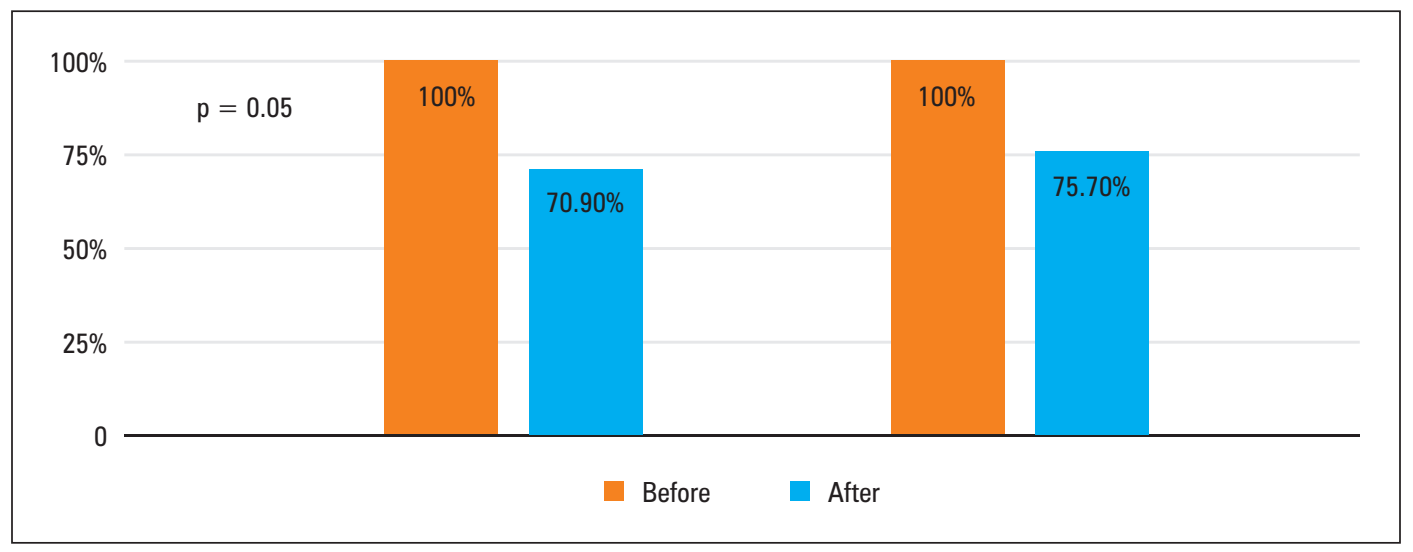

Figure 6. The proportion of patients with insomnia in the groups of combined hypertension with type 2 diabetes mellitus and isolated hypertension after third period of study

there are very little data in the literature on the causes for the development of such a sleep disorder in this group of patients.

Certainly, the development of insomnia in patients with hypertension and type 2 diabetes mellitus is based on several general mechanisms of pathogenesis. First of all, the possible activation of the sympathoadrenal system underlying both the pathogenesis of hypertension and always accompanying insomnia should be taken into account [16, 17]. Markers of systemic inflammation were found to increase both in chronic insomnia as well as in hypertension and type 2 diabetes mellitus. An increase in the production and release of aldosterone was found in patients with insomnia. Also, there are studies demonstrating endothelial dysfunction in chronic sleep disorders. An important factor contributing to the development of insomnia in patients with hypertension and diabetes mellitus is increased anxiety and depression, which also often accompany both conditions. Certainly, the most interesting for the practicing physician is the question: can antihypertensive therapy contribute to the development of insomnia or, on the contrary, reduce the risk of this condition. When searching the PubMed, Clinicaltrials.org, MedLine, SCOPUS databases, we did not find clinical randomized trials that take into account the risk of developing insomnia in hypertensive patients under the influence of antihypertensive therapy. Quite interesting is the paper of Tanabe [18], which is a pharmacoeconomic study. The authors demonstrated that the risk of developing insomnia in patients with hypertension is increased in the highest degree by therapy with alpha- and beta-blockers. So, according to the study by Tanabe [18] the risk of developing insomnia with the administration of alpha-blockers was $\mathrm{OR}=2.38$; 
95\% confidence interval (CI): 1.14-4.98, and in the case of $\beta$-blockers - OR $=1.54$; 95\% CI: 0.99-2.39. In comparison, the risk of developing insomnia with calcium antagonist therapy was $0.67 ; 95 \% \mathrm{CI}$ : $0.47-0.96$. ACE inhibitor therapy was not associated with the risk of developing insomnia. According to the authors' data, insomnia, as a rule, manifested itself by the $5^{\text {th }}$ week of therapy and in more than $50 \%$ of cases manifested itself as "difficulty falling asleep" [18]. A study by Chang [19] also confirmed the ability of beta $\beta$-blockers to induce insomnia and this effect depended on the degree of $\beta(1)$-receptor selectivity and lipophilicity of the drug. Interesting results are presented by Finnish scientists. Haaramo [20] showed that antihypertensive therapy could contribute to the development of insomnia symptoms: OR $=1.57,95 \%$ CI: 1.23-2.00. John W Winkelman [21] demonstrated that diuretic therapy can negatively affect sleep quality. There are also data in literature, which demonstrates an increase in the risk of developing insomnia in the case of using ACE inhibitors through the possibility of developing such an adverse effect as a cough [22]. Nevertheless, the described works are non-systemic and are not prospective studies. Although the abovementioned literature data confirm the presence of a connection between antihypertensive therapy and the risk of developing insomnia, our results demonstrate that the risk of developing insomnia can be reduced by replacing the diuretic indapamide with amlodipine. Our study was conducted as a prospective one with a quite long follow-up period - 12 months.

It should be noted that a decrease in insomnia itself contributes to a decrease in blood pressure [11, 23]. Therefore, a search for relationships between antihypertensive therapy and the risk of developing sleep disorders will allow developing an individualized approach to patients with hypertension and insomnia.

\section{Conclusion}

Correction of antihypertensive therapy, namely the replacement of indapamide with amlodipine, contributes to an improvement in blood pressure, quality of life, and a reduction in the proportion of patients with insomnia.

\section{References}

1. Vgontzas AN, Liao D, Pejovic S, et al. Insomnia with short sleep duration and mortality: the Penn State cohort. Sleep. 2010; 33(9): 1159-1164, doi: 10.1093/sleep/33.9.1159, indexed in Pubmed: 20857861.
2. Eguchi K, Pickering TG, Schwartz JE, et al. Short sleep duration as an independent predictor of cardiovascular events in Japanese patients with hypertension. Arch Intern Med. 2008; 168(20): 2225-2231, doi: 10.1001/archinte.168.20.2225, indexed in Pubmed: 19001199.

3. Patel SR, Ayas NT, Malhotra MR, et al. A prospective study of sleep duration and mortality risk in women. Sleep. 2004; 27(3): 440-444, doi: 10.1093/sleep/27.3.440, indexed in Pubmed: 15164896.

4. Kronholm E, Laatikainen T, Peltonen M, et al. Self-reported sleep duration, all-cause mortality, cardiovascular mortality and morbidity in Finland. Sleep Med. 2011; 12(3): 215-221, doi: 10.1016/j. sleep.2010.07.021, indexed in Pubmed: 21317033.

5. Taylor DJ, Mallory LJ, Lichstein KL, et al. Comorbidity of chronic insomnia with medical problems. Sleep. 2007; 30(2): 213-218, doi: 10.1093/sleep/30.2.213, indexed in Pubmed: 17326547.

6. Jarrin DC, Alvaro PK, Bouchard MA, et al. Insomnia and hypertension: a systematic review. Sleep Med. 2017; 40: e147, doi: 10.1016/j.sleep.2017.11.430, indexed in Pubmed: 29576408.

7. Li M, Yan S, Jiang S, et al. Relationship between sleep duration and hypertension in northeast China: a cross-sectional study. BMJ Open. 2019; 9(1): e023916, doi: 10.1136/bmjopen-2018-023916, indexed in Pubmed: 30670514.

8. Fernandez-Mendoza J, Vgontzas AN, Liao D, et al. Insomnia with objective short sleep duration and incident hypertension: the Penn State Cohort. Hypertension. 2012; 60(4): 929-935, doi: 10.1161/HYPERTENSIONAHA.112.193268, indexed in Pubmed: 22892811.

9. Lin CL, Liu TC, Lin FH, et al. Association between sleep disorders and hypertension in Taiwan: a nationwide population-based retrospective cohort study. J Hum Hypertens. 2017; 31(3): 220-224, doi: 10.1038/jhh.2016.55, indexed in Pubmed: 27511477.

10. Wang YM, Song M, Wang R, et al. Insomnia and Multimorbidity in the Community Elderly in China. J Clin Sleep Med. 2017; 13(4): 591-597, doi: 10.5664/jcsm.6550, indexed in Pubmed: 28212690.

11. Bathgate CJ, Fernandez-Mendoza J. Insomnia, Short Sleep Duration, and High Blood Pressure: Recent Evidence and Future Directions for the Prevention and Management of Hypertension. Curr Hypertens Rep. 2018; 20(6): 52, doi: 10.1007/s11906-0180850-6, indexed in Pubmed: 29779139.

12. Petrov ME, Howard VJ, Kleindorfer D, et al. Over-the-counter and prescription sleep medication and incident stroke: the REasons for Geographic and Racial Differences in Stroke study. J Stroke Cerebrovasc Dis. 2014; 23(8): 2110-2116, doi: 10.1016/j. jstrokecerebrovasdis.2014.03.025, indexed in Pubmed: 25113086.

13. Williams B, Mancia G, Spiering W, et al. 2018 ESC/ESH Guidelines for the management of arterial hypertension. J Hypertens. 2018; 36(10): 1953-2041, indexed in Pubmed: 30234752.

14. Unifikovanyi klinichnyi protokol pervynnoi ta vtorynnoi (spetsializovanoi) medychnoi dopomohy: tsukrovyi diabet 2 typu (nakaz MOZ 1118 vid 21.12.2012 r. [In Ukrainian].

15. American Academy of Sleep Medicine. International classification of sleep disorders, 3rd ed. American Academy of Sleep Medicine, Darien 2014.

16. Carter JR, Grimaldi D, Fonkoue IT, et al. Assessment of sympathetic neural activity in chronic insomnia: evidence for elevated cardiovascular risk. Sleep. 2018; 41(9), doi: 10.1093/sleep/ zsy126, indexed in Pubmed: 29992317.

17. Tamisier R, Weiss JW, Pépin JL. Sleep biology updates: Hemodynamic and autonomic control in sleep disorders. Metabolism. 2018; 84: 3-10, doi: 10.1016/j.metabol.2018.03.012, indexed in Pubmed: 29572132.

18. Tanabe N, Fujita T, Fujii Y, et al. [Investigation of the factors that contribute to the onset of insomnia in hypertensive patients by using a post-marketing surveillance database]. Yakugaku Zasshi. 2011; 131(5): 669-677, doi: 10.1248/yakushi.131.669, indexed in Pubmed: 21532263.

19. Chang CH, Yang YHK, Lin SJ, et al. Risk of insomnia attributable to $\beta$-blockers in elderly patients with newly diagnosed hypertension. 
Drug Metab Pharmacokinet. 2013; 28(1): 53-58, doi: 10.2133/ dmpk.dmpk-12-rg-004, indexed in Pubmed: 22813717.

20. Haaramo P, Rahkonen O, Hublin C, et al. Insomnia symptoms and subsequent cardiovascular medication: a register-linked follow-up study among middle-aged employees. J Sleep Res . 2014; 23(3): 281-289, indexed in Pubmed: 24313664.

21. Winkelman JW, Benca R, Eichler AF. Overview of the treatment of insomnia in adults. https://www.uptodate.com/contents/ overview-of-the-treatment-of-insomnia-in-adults.
22. Sato A, Fukuda S. A prospective study of frequency and characteristics of cough during ACE inhibitor treatment. Clin Exp Hypertens. 2015; 37(7): 563-568, doi: 10.3109/10641963.20 15.1026040, indexed in Pubmed: 25992489.

23. Lu Y, Wang X, Yang G, et al. Effects of Chronic Intractable Insomnia on Inflammatory Cytokines, Blood Pressure Characteristics, and Antihypertensive Efficacy in Hypertensive Patients. Med Sci Monit. 2018; 24: 9259-9264, doi: 10.12659/MSM.911997, indexed in Pubmed: 30568156. 